\title{
SÍNDROME HELLP: RESULTADO MATERNO FETAL. UNIDAD DE CUIDADOS INTENSIVOS MATERNO DEL INSTITUTO NACIONAL MATERNO PERINATAL
}

\author{
Walter Jerry De la Peña Meniz¹, Alberto Martín Díaz Seminario ${ }^{1}$, Ronald Meza Salcedoㄹ, \\ Hernán Sandoval Manrique ${ }^{1}$, Julio César Cano Loayza ${ }^{1}$, Marco Antonio Delgado Fuentes ${ }^{1}$, \\ Franklin Mendoza Torres ${ }^{1}$, Kenny Eli Villalobos Corrales ${ }^{1}$, José Augusto Juarez Silva ${ }^{1}$, \\ Alfredo Castillo Goz $r^{1}$, José luis Castillo Lino ${ }^{1}$, Lorena Manrique Montoya ${ }^{1}$, Xandra Rodriguez Tucto ${ }^{1}$.
}

\begin{abstract}
RESUMEN
Objetivo. conocer las características epidemiológicas clínicas y patológicas del Síndrome HELLP de pacientes admitidas a la UCIM del INMP. Materiales y métodos. se revisaron las historias clínicas de las pacientes admitidas a la Unidad de Cuidados Intensivos Materno del INMP, desde agosto del año 2014 hasta el 31 de agosto del año 2016. Ademas, se incluyeron 502 registros; 49 registros de pacientes con diagnóstico de síndrome HELLP y 452 registros de pacientes con diagnóstico de preeclampsia. Resultados. La media de edad fue de $31.27+/-7.30$ años (p:0.000), la edad de inicio de actividad sexual fue de 18.2+/-3.31 años (0.0004), la media de EG fue de 34.05+/-5.19 semanas, las medias de TGO y TGP fueron 521.06 U/L (p:0.000) y $599.78 \mathrm{U} / \mathrm{L}(\mathrm{p}: 0.000)$, respectivamente, la media de plaquetas fue de $58619+/-28938$, la PAS fue mayor en el grupo de pacientes con síndrome HELLP; $139.11+/-24.01 \mathrm{mmHg}$ (p:0.000). Conclusiones. El síndrome HELLP posee factores de riesgo ya determinados que permiten sospechar la nueva aparición de los síntomas en una mujer gestante. La mayor frecuencia de HELLP se produce a mayor edad, a mayor edad de inicio de actividad sexual. La Presión arterial sistólica es mayor en pacientes con Síndrome de HELLP que en otras pacientes con trastorno hipertensivo del embarazo.
\end{abstract}

Palabras claves: Síndrome de HELLP, trastornos hipertensivos del embarazo (Fuente DeCS BIREME).

\section{HELLP SYNDROME: FETAL MATERNAL RESULT. MATERNAL INTENSIVE CARE UNIT OF THE PERINATAL MATERNAL NATIONAL INSTITUTE}

\begin{abstract}
Objective. Know clinical and pathological epidemiologic characteristics from HELLP Syndrome of admitted patients in the Maternal Intensive Care Unit from INMP. Materials and methods. Clinical histories from admitted patients in the Maternal Intensive Care Unit from INMP since August of 2014 to August $31^{\text {st }}$ of 2016 were checked out. Furthermore, 502 records: 49 record of patients with HELLP Syndrome y 452 records of patients with preeclampsia diagnostic were included. Results. The average age was $31.27+/-7.30$ years old ( $p: 0.000$ ), the age of initiation of sexual activity was $18.2+/-3.31$ years old (0.0004), the average of gestational age was $34.05+/-5.19$ weeks, the average of ALT and AST were 521.06 U/L (p:0.000) y $599.78 \mathrm{U} / \mathrm{L}$ (p:0.000), respectively, the average of platelets was $58619+/-28938$ and SBP was the main in the patients with HELLP Syndrome group with $139.11+/-24.01 \mathrm{mmHg}$ ( $\mathrm{p}: 0.000$ ). Conclusions. The HELLP syndrome has determined risk factors that allow suspecting the new onset of symptoms in a pregnant woman. The greater frequency of HELLP occurs at older age, the older the age of onset of sexual activity. Systolic blood pressure is higher in patients with HELLP syndrome than in other patients with hypertensive disorder of pregnancy.
\end{abstract}

Key words: HELLP syndrome, hypertensive disorders of pregnancy (Source: MeSH NLM).

\section{INTRODUCCIÓN}

El Síndrome HELLP acuñado por Louis weinstein el año de 1982, es una trilogía (hemolisis, transaminasemia y disminución del número de plaquetas) que complica a la paciente con Preeclampsia, se presenta por encima de las 20 semanas de gestación y conlleva una serie de complicaciones materno fetales.

El año de 1982 Weinstein publicó en el American Journal Obstetric and Gynecology, en ella catalogo un grupo único de pacientes preeclampticas /eclampticas con el hallazgo de Hemolisis, elevación de enzimas hepáticas y plaquetopenia.a las cuales se acuño con el acrónimo de HELLP ${ }^{(1)}$.

Las complicaciones asociados a este síndrome comprometen no solo a la madre sino también al feto; las complicaciones maternas pasan por un incremento en la morbimortalidad materna, la presencia de rotura hepática espontanea, incremento en la frecuencia de insuficiencia renal, coagulopatia asociado a la trombocitopenia y mayor riesgo de sangrado, así como mayor frecuencia de desprendimiento prematuro de placenta ${ }^{(2)}$.

Médico Intensivista. Unidad de Cuidados Intensivos Maternos. Instituto Nacional Materno Perinatal. Lima-Perú. 
La frecuencia de presentación es mayor de las 37 semanas de gestación, un $70 \%$ de las pacientes presenta síndrome HELLP en el anteparto y un $30 \%$ en el post parto ${ }^{(3)}$.

Un grupo de pacientes porcentualmente menor presenta el síndrome a edad gestacional menor de 26 semanas, en condiciones de mucho riesgo para la sobrevivencia del feto. Lo cual hace necesario buscar formas de tratamiento basado en la identificación temprana de pacientes en riesgo de hacer Síndrome HELLP. Para evitar el riesgo de prematuridad y mortalidad fetal ${ }^{(4)}$.

\section{MATERIALES Y MÉTODOS}

El presente corresponde a un estudio descriptivo transversal comparativo, se revisaron las historias clínicas de las pacientes admitidas a la Unidad de Cuidados Intensivos Materno del INMP, desde Agosto del año 2014 hasta el 31 de agosto del año 2016. Se incluyeron 502 registros ; 49 registros de pacientes con diagnóstico de síndrome HELLP y 452 registros de pacientes con diagnóstico de preeclampsia. No se tomaron en cuenta registros con diagnóstico de rotura hepática, Las variables cuantitativas edad, peso, talla, IMC, menarquia, edad de primera RS, edad gestacional, peso del recién nacido (RN), Talla del RN, APGAR al minuto $y$ a los 5 minutos, hemoglobina, TGO, TGP, plaquetas, PAS, Frecuencia Cardiaca, Creatinina, y estancia hospitalaria. Las variables cualitativas estado civil, primipaternidad, antecedente de HIE, antecedente de síndrome HELLP, condición de ingreso a la UCIM (referida o demanda interna), cesárea, uso de hemoderivados, la edad reagrupada y la edad gestacional reagrupada. Las variables cuantitativas previa evaluación de normalidad se compararon obteniendo el $p$ valor, las variables cualitativas se compararon mediante tablas de frecuencia. Se aplicaron pruebas paramétricas o no paramétricas según evaluación previa de normalidad y se usó una significación estadística $p<0,05$. el análisis estadístico se realizó utilizando el paquete estadístico STATA versión 13.

\section{RESULTADOS}

En la tabla 1 se observa que la variable Estado civil de convivencia o unión estable fue la más frecuente (61.70\%), respecto a la primipaternidad se presentó en las pacientes con síndrome HELLP en un $34.5 \%, 19.57 \%$ y $17.24 \%$ tenían antecedentes de hipertensión inducida por el embarazo y HELLP en embarazaos previos. $76.19 \%$ de las pacientes fueron referidas de otros establecimientos. $86.67 \%$ culminaron el embarazo por cesárea. $11.11 \%$ recibieron transfusión de hemoderivados, el grupo etareo más frecuente fue de 25-34 años de edad (48.98\%), la edad gestacional más frecuente fue de 36-42 semanas. El nivel de plaquetas más frecuentes fue menor de 50 mil $(54.76 \%)$.

En la Tabla 2 observamos que de 502 gestantes, 49 presentaron síndrome HELLP y 452 registros de pacientes con diagnóstico de preeclampsia. Se excluyeron del estudio las pacientes con diagnóstico de shock hemorrágico y rotura hepática. En las pacientes con diagnóstico de HELLP la edad promedio correspondió a $31.27+/-7.30$ años de edad, el peso promedio $62.07+/$ 10.82 , la talla promedio fue de $1.53+/-0.62$, el promedio de IMC fue de $24.42+/-8.13$, el promedio de menarquia fue de $13.02+/-1.27$, el promedio de la edad de primera relación sexual fue de $18.2+/-3.31$ años, el promedio de edad gestacional fue de $34.05+/-5.19$ semanas, el promedio del peso del RN fue de 2351.18+/- 892.97 grs, el promedio de talla del RN fue de $61.72+/-7.11 \mathrm{~cm}$, el promedio de APGAR al minuto fue de $5.86+/-3.10$, el promedio de APGAR a los 5 minutos fue de $7.03+/-3.46$, el promedio de la hemoglobina materna fue de $10.07+/-2.75$ $\mathrm{gr} / \mathrm{dl}$, el promedio de TGO fue de $521.06+/-32.80 \mathrm{U} / \mathrm{l}$, el promedio de TGP fue de $436.37+/-26.43 \mathrm{U} / \mathrm{l}$, el promedio de plaquetas fue de $58619+/-28900$, el promedio de PAS fue de $139.11+/-24.01 \mathrm{mmHg}$, el promedio de Frecuencia Cardiaca (FC) fue de $85.54+/-16.15 \mathrm{lpm}$, el promedio de Creatinina fue de $2.19+/-1.8 \mathrm{mg} / \mathrm{dl}$, el promedio de estancia hospitalaria fue de $5.29+/-4.17$ dias.

En la tabla 3 se observa que la complicación más frecuente de las pacientes admitidas a la UCIM del INMP fue Insuficiencia Respiratoria de tipo oxigenatoria (36\%), en segundo lugar eclampsia y falla renal con $20 \%$ cada uno y en tercer lugar shock hemorrágico $(12 \%)$

Tabla 1. Comparación de características Sociodemográficas entre gestantes con Síndrome HELLP y gestantes con Trastornos Hipertensivos del Embarazo. Instituto Nacional Materno Perinatal. 2014-2016

\begin{tabular}{lccccc}
\hline \multirow{2}{*}{ VARIABLE } & \multicolumn{3}{c}{ HELLP } & \multicolumn{3}{c}{ THE } \\
\cline { 2 - 6 } & $\mathrm{N}$ & $\%$ & $\mathrm{~N}$ & $\%$ & $\mathrm{P}$ \\
\hline E. CIVIL & & & & & 0.42 \\
-CASADA & 10 & 21.28 & 56 & 12.67 & \\
-CONVIV & 29 & 61.70 & 299 & 67.65 & \\
-SOLTERA & 8 & 17.02 & 86 & 19.46 & \\
-VIUDA & 0 & 0 & 1 & 0.23 & \\
PRIMIPAT & 14 & 34.5 & 181 & 41.5 & 0.173 \\
\hline ANT HIE & 9 & 19.57 & 47 & 10.83 & 0.079 \\
\hline ANT HELLP & 5 & 17.24 & 9 & 3.70 & 0.01 \\
REFERIDA & 32 & 76.19 & 147 & 41.29 & 0.000 \\
\hline CESAREA & 39 & 86.67 & 291 & 89.26 & 0.377 \\
HEMOD & 5 & 11.11 & 23 & 5.52 & 0.176 \\
GRUPOEDAD & & & & & 0.000 \\
14-24 AÑOS & 8 & 16.33 & 199 & 43.83 & \\
25-34 AÑOS & 24 & 48.98 & 181 & 39.87 & \\
35-44 AÑOS & 17 & 34.69 & 74 & 16.30 & \\
EG AGRUP & & & & & 0.002 \\
\hline 20-27 SS & 3 & 7.89 & 84 & 23.86 & \\
28-33 SS & 11 & 28.95 & 99 & 28.13 & \\
\hline 34-35 SS & 10 & 26.32 & 40 & 11.36 & \\
\hline 36-42 SS & 14 & 36.84 & 129 & 36.65 & \\
\hline PLAQUETAS AGRUP & & & & 0.000 \\
\hline < 50 mil & 23 & 54.76 & 51 & 13.78 & \\
\hline 50-100mil & 14 & 33.33 & 74 & 19.27 & \\
\hline 101-150mil & 5 & 11.90 & 67 & 17.45 & \\
\hline
\end{tabular}

Fuente: Registros clínicos del Instituto Nacional Materno Perinatal 


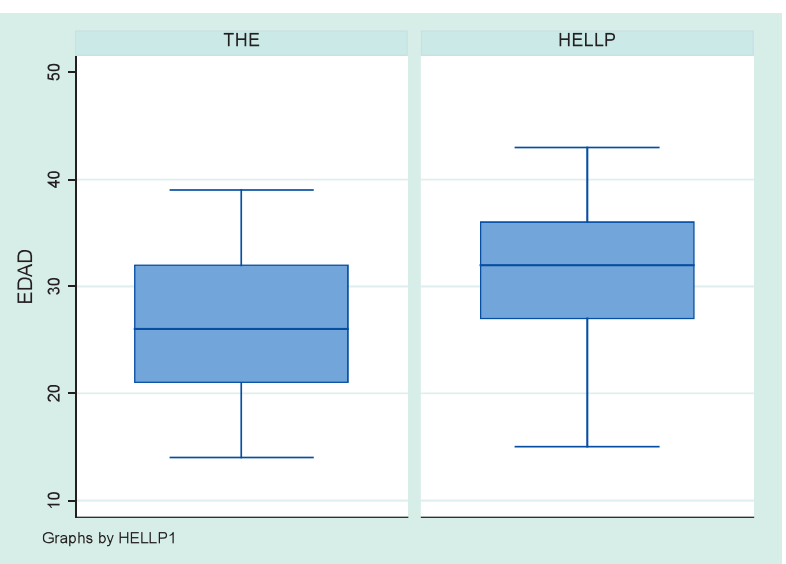

Gráfico 1. Edad materna entre gestantes con síndrome de HELLP y gestantes con trastornos hipertensivos del embarazo. Instituto Nacional Materno Perinatal. 2014-2016

Fuente: Registros clínicos del Instituto Nacional Materno Perinatal

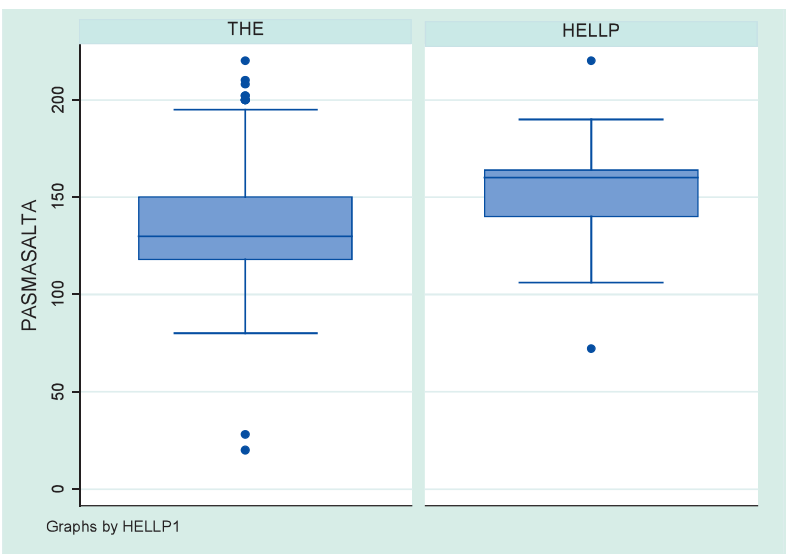

Gráfico 2. Presión Arterial Sistólica entre gestantes con síndrome de HELLP y gestantes con trastornos hipertensivos del embarazo. Instituto Nacional Materno Perinatal. 2014-2016

Fuente: Registros clínicos del Instituto Nacional Materno Perinatal
Tabla 3. Complicaciones más frecuentes asociados a síndrome HELLP. Instituto Nacional Materno Perinatal. 2014-2016

\begin{tabular}{lcc}
\hline COMPLICAC & N & $\%$ \\
\hline ECLAMPSIA & 5 & 20 \\
HIC & 1 & 4 \\
IRA I & 9 & 36 \\
F RENAL & 5 & 20 \\
R HEPATICA & 1 & 4 \\
SDMO & 1 & 4 \\
SHOCK H & 3 & 12 \\
TOTAL & 25 & 100 \\
\hline
\end{tabular}

Fuente: Registros clínicos del Instituto Nacional Materno Perinatal

\section{DISCUSIÓN}

Se revisaron las historias clínicas de las pacientes admitidas a la Unidad de Cuidados Intensivos Materno del INMP, desde Agosto del año 2014 hasta el 31 de agosto del año 2016. Se incluyeron 502 registros; 49 registros de pacientes con diagnóstico de síndrome HELLP y 452 registros de pacientes con diagnóstico de preeclampsia. Al evaluar la variable edad se observan diferencias estadísticamente significativas, observamos que la media de edad de presentación del síndrome HELLP fue mayor que en aquellas pacientes con THE sin HELLP. La edad gestacional de inicio de síndrome HELLP fue maø $r$ respecto de aquellas pacientes con THE sin HELLP. Los valores de creatinina también mostraron diferencias significativas, siendo valores mayores en las pacientes con síndrome HELLP. La estancia hospitalaria fue mayor en la pacientes con síndrome HELLP, dato esperable por las complicaciones adicionales que presentan este grupo de pacientes y que retrasa la recuperación de las pacientes. Se encontró diferencias respecto a la edad de

Tabla 2. Comparación de características clínicas entre madres con Síndrome HELLP y madres con Trastornos Hipertensivos del Embarazo. Instituto Nacional Materno Perinatal. 2014-2016

\begin{tabular}{|c|c|c|c|c|c|}
\hline \multirow{2}{*}{ VARIABLE } & \multirow{2}{*}{$\begin{array}{l}\text { HELLP } \\
\text { MEDIA }\end{array}$} & \multicolumn{3}{|c|}{ THE } & \multirow{2}{*}{$\begin{array}{l}P \\
P\end{array}$} \\
\hline & & IC & MEDIA & IC & \\
\hline EDAD & 31.27 & $29.17-33.36$ & 26.65 & $26.05-27.25$ & 0.000 \\
\hline PESO & 62.07 & $58.61-65.53$ & 61.70 & $60.56-62.83$ & 0.844 \\
\hline TALLA & 1.53 & $1.51-1.55$ & 1.54 & $1.53-1.54$ & 0.663 \\
\hline IMC & 24.42 & $21.62-27.22$ & 25.34 & $24.63-26.05$ & 0.28 \\
\hline MENARQUIA & 13.02 & $12.62-13.43$ & 12.78 & $12.66-12.89$ & 0.23 \\
\hline EDAD $1^{\circ} \mathrm{RS}$ & 18.2 & $17.14-19.26$ & 16.85 & $16.6471 \quad 17.07$ & 0.0004 \\
\hline EG & 34.05 & $32.35-35.76$ & 30.94 & $30.04-31.83$ & 0.028 \\
\hline PESO RN & 2351.18 & 2004.92-2697.43 & 2474.56 & 2365.982583 .14 & 0.50 \\
\hline TALLA RN & 61.72 & $25.67-97.78$ & 80.49 & $66.49-94.50$ & 0.426 \\
\hline APGAR MIN & 5.86 & $4.68-7.04$ & 6.34 & $6.03-6.65$ & 0.299 \\
\hline APGAR5MIN & 7.03 & $5.72-8.35$ & 7.64 & 7.33-7.94 & 0.82 \\
\hline HB & 10.07 & $9.29-10.86$ & 9.81 & $9.57-10.05$ & 0.50 \\
\hline TGO & 521.063 & $325.23-716.89$ & 130.35 & $89.83-170.87$ & 0.000 \\
\hline TGP & 436.37 & $262.22-610.53$ & 104.05 & $68.76-139.35$ & 0.000 \\
\hline PLAQUETAS & 58619 & $49601-67637$ & 155019 & & 0.000 \\
\hline PAS & 139.11 & $131.89-146.32$ & 117.86 & $115.28-120.43$ & 0.000 \\
\hline FC & 85.54 & $80.75-90.34$ & 95.65 & $93.57-97.72$ & 0.0019 \\
\hline CREAT & 2.19 & $1.11-3.26$ & 1.57 & $1.35-1.79$ & 0.0195 \\
\hline ESTANCIA & 5.29 & $3.76-6.82$ & 4.32 & $3.77-4.90$ & 0.0463 \\
\hline
\end{tabular}

Fuente: Registros clínicos del Instituto Nacional Materno Perinatal 
primera relación sexual; la frecuencia de Síndrome HELLP fue may $r$ cuando la edad de inicio de actividad sex al fue mayor. Las transaminasa y el recuento de plaquetas también mostro diferencias significativas hallazgo esperado pues es una característica del Síndrome HELLP. La media de PAS también mostro diferencias significativas a favor del síndrome HELLP. Hallazgo que debería confirmarse en estudios de cohorte prospectivo. Respecto a las variables cualitativas un $17.4 \%$ de pacientes referían antecedente de HELLP en un embarazo previo ( $p$ : 0.01 ), un $76.19 \%$ de las paciente admitidas con diagnóstico de síndrome HELLP fueron referidas de otros establecimientos de salud ( $\mathrm{p}:$ 0.000). La edad promedio de pacientes que presentaron síndrome HELLP fue de 25 a 34 años de edad, diferencia que fue estadísticamente significativa ( $p: 0.000$ ). la edad gestacional de presentación del síndrome HELLP más frecuente fue entre 36 a 42 semanas de gestación (36.84\%, p:0.002). Respecto a la trombocitopenia el grupo menor de 50 mil plaquetas fue estadísticamente significativa (54.76\% p: 0.000).

\section{CONCLUSIONES}

El síndrome HELLP tiene importancia por la implicancia sobre la morbimortalidad materna, posee factores de riesgo ya determinados que permiten sospechar la nueva aparición de los síntomas en una mujer gestante. Falta aún determinar los factores pronóstico que permitan determinar las probabilidades de riesgo. Es importante notar algunos resultados del presente estudio; como la may $r$ frecuencia de HELLP cuanto maø $r$ sea la edad de la paciente, a mayor edad de inicio de actividad sexual mayor riesgo de HELLP. En grupo de estudio se encontró que presentaban una media de Presion arterial sistólica mayor que el grupo control y finalmente que el mayor porcentaje de pacientes con síndrome HELLP fueron admitidas con medias de trombocitopenia menores de 50 mil plaquetas.

Se requieren de estudios de cohorte tipo prospectivo para confirmar los datos obtenidos del presente estudio. Es necesario poder evaluar algunas otras variables que nos permitan tener una idea global de la forma de presentación de las pacientes admitidas en nuestra institución.

Financiamiento: Autofinanciado.

Conflicto de interés: Los autores declaran no tener algún conflicto de intereses.

\section{REFERENCIAS BIBLIOGRÁFICAS}

1. Weinstein L. Syndrome of hemolysis, elevated liver enzymes, and low platelet count: a severe consequence of hypertension in pregnancy. Am J Obstet Gynecol. 15 de enero de 1982;142(2):159-67.

2. Sibai BM, Taslimi MM, El-Nazer A, Amon E, Mabie BC, Ryan GM. Maternal-perinatal outcome associated with the syndrome of hemolysis, elevated liver enzymes, and low platelets in severe preeclampsia-eclampsia. Am J Obstet Gynecol. 1 de septiembre de 1986;155(3):501-7.

3. Georgiew DB, Skortcheva I, Abadjiew V. Early development of HELLP syndrome: a case report. Gynecol Obstet Invest. 1990;30(2):127-8.

4. van Oostwaard $M$, van Eerden $L$, de Laat $M$, Duvekot J, Erwich J, Bloemenkamp K, et al. Maternal and neonatal outcomes in women with severe early onset pre-eclampsia before 26 weeks of gestation, a case series. BJOG Int J Obstet Gynaecol. 1 de agosto de 2017;124(9):1440-7.

Correspondencia: $d$. Santa Rosa 941. Lima 1.

Teléfono: 996014918

E-mail:wdlpmeniz@gmail.com

\section{ORCID iDs}

Walter Jerrv De la Peña Meniz Ronald Meza Salcedo

Marco Antonio Delgado Fuentes httbs://orcid ora/0000-0002-3261-773X https://orcid.org/0000-0001-9707-6519

https://orcid.org/0000-0003-4254-3452 